\title{
Blind image quality assessment through anisotropy
}

\author{
Salvador Gabarda and Gabriel Cristóbal* \\ Instituto de Óptica "Daza de Valdés" (CSIC), Serrano 121, Madrid 28006, Spain \\ *Corresponding author: gabriel@optica.csic.es
}

Received February 26, 2007; revised July 9, 2007; accepted July 18, 2007; posted July 25, 2007 (Doc. ID 80401); published September 26, 2007

\begin{abstract}
We describe an innovative methodology for determining the quality of digital images. The method is based on measuring the variance of the expected entropy of a given image upon a set of predefined directions. Entropy can be calculated on a local basis by using a spatial/spatial-frequency distribution as an approximation for a probability density function. The generalized Rényi entropy and the normalized pseudo-Wigner distribution (PWD) have been selected for this purpose. As a consequence, a pixel-by-pixel entropy value can be calculated, and therefore entropy histograms can be generated as well. The variance of the expected entropy is measured as a function of the directionality, and it has been taken as an anisotropy indicator. For this purpose, directional selectivity can be attained by using an oriented 1-D PWD implementation. Our main purpose is to show how such an anisotropy measure can be used as a metric to assess both the fidelity and quality of images. Experimental results show that an index such as this presents some desirable features that resemble those from an ideal image quality function, constituting a suitable quality index for natural images. Namely, infocus, noise-free natural images have shown a maximum of this metric in comparison with other degraded, blurred, or noisy versions. This result provides a way of identifying in-focus, noise-free images from other degraded versions, allowing an automatic and nonreference classification of images according to their relative quality. It is also shown that the new measure is well correlated with classical reference metrics such as the peak signal-to-noise ratio. (C) 2007 Optical Society of America

OCIS codes: $110.3000,100.2000,330.6180,100.6640$.
\end{abstract}

\section{INTRODUCTION}

The objective of a great number of applications of image processing is the search for an in-focus, noise-free version of an unknown original. Image quality assessment (QA) is not a minor issue, especially if more than one enhanced image competes to be the best realization. The naked eye is surely a good classifier, but an automatized method would save time and should simplify the task. Quality evaluation of images is still an open and challenging problem [1]. Typically, methods for determining the quality of enhanced images [2,3] require a reference or ground truth image to fulfill the measure. Measures such as peak signal-to-noise ratio (PSNR) or root-mean-squared error (RMSE) are simple to calculate, but they are not always in agreement with the perceived visual quality. Significant improvement has been attained by some image QA methods that take advantage of known characteristics of the human visual system (HVS); for example, Wang and Bovik [4] describe a method based on the hypothesis that the HVS is highly adapted for extracting structural information, and they develop a measure of structural similarity (SSIM) that compares local patterns of pixel intensities that have been normalized for luminance and contrast. Sheikh et al. [5] use an information fidelity criterion (IFC) for image QA using natural scene statistics models as an alternative to the HVS-based methods. In another paper [6], Sheikh et al. explore the relationship between image information and visual quality, and they introduce a visual information fidelity (VIF) criterion for full-reference image QA. The VIF is derived from a statis- tical model for natural scenes, a model for image distortions, and a HVS model in an information-theoretic setting. However, when a reference image is not available, only blind image QA methods may be useful. Sheikh et al. [7] propose the use of natural scene statistics (NSS) models to blindly measure the quality of images compressed by JPEG2000 as a novel way of assessing the quality of images afflicted with ringing and blurring distortion resulting from JPEG2000 compression. Remarkably, this method uses NSS models to provide a reference against which the distorted images can be assessed.

A new image QA method that does not require a reference image to determine the quality of the images under scrutiny is introduced here. This blind image QA method is based on measuring the anisotropy of the images and has been confirmed to be robust over an ample set of images and akin to the way the HVS works. Hence, the automatized selection of the "best image" from a set of possibilities coincides well with the criteria of the HVS, as the experiments presented here will later corroborate, with a set of natural images.

Shannon entropy is classically used as a value to indicate the amount of uncertainty or information in a source [8]. Quality and entropy are somewhat related subjects. If the source is a given image, the obstacle for the entropy to be considered a quality index is that noise cannot be distinguished from information, noise being a kind of information itself. From a human observer point of view, objects constitute the areas of interest in a picture, and humans with good eye correction are easily capable of dis- 
tinguishing the sharpest objects. Noise or blurring is easily identifiable by the visual system. Analytically, entropy increases with sharpness but, in general, there is not a fair correlation when images are noisy. Hence, entropy by itself is not a good indicator of image quality. To overcome this problem, in this paper we propose to use anisotropy as a suitable measure of image quality.

Based on our experiments, we hypothesize that the degradation processes damage the scene's directional information. Hence, anisotropy, as a directionally dependent quality of images, decreases as more degradation is added to the image.

There exists an antecedent of the use of entropy for identifying the anisotropy of images [9]. Anisotropy is certainly one of the properties of natural images and is related to its directional dependency. Our experiments have shown that image anisotropy is sensitive to noise and blur; hence quality can be properly measured in this way. Following this line of reasoning, we have extended the applications of entropy to the problem of measuring image quality in a nonreference scenario. That is, following this approach no reference is required to sort images according to their visual quality. The generalized Rényi entropy has been selected to calculate the entropy on a local basis by associating a distribution for each pixel of a digital image. In this way, entropy histograms provide a measure of the information content of images in the same way as image histograms give information about the distribution of gray levels. Using a proper normalization, a windowed pseudo-Wigner distribution (PWD) can be approximated as a probability distribution function [10], and then a particular Rényi-wise entropy can be measured at the pixel level. This PWD is computed in a 1-D-oriented window, allowing a measure of the entropy in a selected direction. Differences in the directional entropy are taken to measure image anisotropy and hence to estimate of the image quality. Different techniques have been proposed in the literature for assessing images when the ground truth is not available [11-14]. In the context of image QA, Dansereau and Kinser were the first to suggest the potential use of the relative Rényi dimension spectrum as an image quality measure [15]. The main motivation of this paper is to provide an in-depth study on the use of highorder Rényi entropies in the area of nonreference image quality evaluation.

This paper is structured as follows: The basic mathematical description and theoretical fundamentals of the method are described in Section 2. Section 3 presents the new quality metric and its empirical justification. Section 4 presents experimental results obtained by applying this method to some artificial and real examples. Finally, conclusions are drawn in Section 5.

\section{MATHEMATICAL BACKGROUND}

\section{A. Rényi Entropy Measures}

Entropy is a measure of the information content of given data. In general, any 2-D array that entails information can be considered an image. This suggests that differences in entropy orientations can provide differences in the information content. That is, information can be stored in an anisotropic way. Entropy can be applied as a global measure or as a local one, adding the possibility of different directionalities when dealing with images. Entropy is an important feature to consider, especially when orientation inherits some specific information. Subsequently, directional entropy measurements can be used to determine differences between different images or textures with different entropic characteristics. Directional entropy can be achieved by means of the Rényi entropy. For this reason, Rényi entropy measurements stand out as a relevant entropic measure in this context.

A review of the existing Rényi measures, which we summarize here, can be found in [16]. The definition of entropy was initially proposed independently by Shannon [8] and Wiener [17] as a measure of the information content per symbol, coming from a stochastic information source. Later, Rényi [18] extended this notion to yield generalized entropy. Different distributions have been considered to define some Rényi entropy measures. They have been introduced in the time-frequency analysis area by Williams et al. [19], with a significant contribution by Flandrin et al. [20], establishing the properties of such measures. In general, the Rényi entropy applied to a discrete space-frequency distribution $P[n, k]$ has the form

$$
R_{\alpha}=\frac{1}{1-\alpha} \log _{2}\left(\sum_{n} \sum_{k} P^{\alpha}[n, k]\right)
$$

It is interesting to note that the Shannon entropy given by

$$
H=-\sum_{n} \sum_{k} P_{x}[n, k] \log _{2}\left(P_{x}[n, k]\right)
$$

can be obtained from the Rényi entropy measure in the limiting case when $\alpha \rightarrow 1$ [20].

Here $n$ and $k$ represent the spatial and frequency variables, respectively. Also, $\alpha \geqslant 2$ are values recommended for space-frequency distribution measures [20]. Although Rényi measures of joint space-frequency distributions formally look like the original entropies, they do not have the same properties, conclusions, and results derived in classical information theory. For instance, the positivity $P[n, k] \geqslant 0$ will not be always preserved, along with the unity energy condition $\Sigma_{n} \Sigma_{k} P[n, k]=1$. In order to reduce a distribution to the unity signal energy case, some kind of normalization must be done [19]. The normalization can be done in various ways, leading to a variety of different measure definitions $[16,20]$.

\section{Normalization with the Signal Energy}

$$
R E_{\alpha}=\frac{1}{1-\alpha} \log _{2}\left(\frac{\sum_{n} \sum_{k} P^{\alpha}[n, k]}{\sum_{n} \sum_{k} P[n, k]}\right) \text { with } \alpha \geqslant 2 .
$$

The behavior of this measure is quite similar to the nonnormalized measure form, except in its magnitude. This kind of normalization is important for comparison between various distributions, or with the same distribution when the energy is not unbiased. 


\section{Normalization with the Distribution Volume}

$$
R V_{3}=-\frac{1}{2} \log _{2}\left(\frac{\sum_{n} \sum_{k} P^{3}[n, k]}{\sum_{n} \sum_{k}|P[n, k]|}\right)
$$

The volume-normalized form of measure has been used for adaptive kernel design [19]. Note that the term within the logarithm is just the ratio of norms $L_{3}$ and $L_{1}$, while the logarithm is a monotonic function. Thus, measure (4) can be considered to be $L_{3} / L_{1}$, reducing to the general case.

\section{Quantum Normalization}

Quantum mechanics [21] inspires a normalization by assimilating the spatial/spatial-frequency distribution $P$ of a given position $n$ with a wave function and deriving its probability density function by means of $\breve{P}[n, k]$ $=P[n, k] P^{*}[n, k]$, followed by a normalizing step to satisfy the condition $\Sigma_{n} \Sigma_{k} \breve{P}[n, k]=1$.

The general case in expression (1) with $\alpha=3$ gives

$$
\breve{R}_{3}=-\frac{1}{2} \log _{2}\left(\sum_{n} \sum_{k} \breve{P}^{3}[n, k]\right) .
$$

This measure can be interpreted on a pointwise basis as follows:

$$
\breve{R}_{3}[n]=-\frac{1}{2} \log _{2}\left(\sum_{k} \breve{P}^{3}[n, k]\right) .
$$

The term $\breve{P}$ in expression (6) also has to be normalized by $Q[n, k]=P[n, k] P^{*}[n, k], \quad$ followed by $\breve{P}[n, k]$ $=Q[n, k] / \Sigma_{k} Q[n, k]$ to meet the normalizing condition: $\Sigma_{k} \breve{P}[n, k]=1, \forall n: 1 \leqslant n \leqslant M$, where $M$ is the size of the data and $-N / 2 \leqslant k \leqslant N / 2-1$ the spatial window used to compute the measure. We have selected this normalization in particular in the sequel.

\section{B. One-Dimensional Pseudo-Wigner Distribution}

Spatial-frequency information of a given image can be extracted by associating the gray-level spatial data with one of the well-known spatial/spatial-frequency distributions [22]. Typically, the Rényi entropy is applied over one of these joint distributions. In this paper the Wigner distribution has been selected [23] due to its excellent properties. In such case, any specific pixel $n$ of the image can be associated to a vector containing its 1-D PWD, calculated in a neighborhood by means of a small window of length $N$. The use of a windowed 1-D transform for a 2-D signal can be justified considering three main aspects of the problem. First, by using a 1-D PWD, data can be arranged in any desired direction over a 2-D image; second, calculation time is greatly diminished compared to a $2-\mathrm{D}$ version of the PWD; and third, the 1-D PWD is an invertible function, granting in this way that information is totally preserved.

A discrete approximation of the Wigner distribution proposed by Claasen and Mecklembräuker [24], similar to Brenner's expression [25], has been used here:

$$
W_{z}[n, k]=2 \sum_{m=-N / 2}^{N / 2-1} z[n+m] z^{*}[n-m] e^{-2 i(2 \pi m / N) k} .
$$

In Eq. (7), $n$ and $k$ represent the time and frequency discrete variables, respectively, and $m$ is a shifting parameter, which is also discrete. Here $\{z[n]\}$ is a $1-\mathrm{D}$ sequence of data from the image, containing the gray values of $N$ pixels, aligned in the desired direction. Equation (7) can be interpreted as the discrete Fourier transform (DFT) of the product $z[n+m] z^{*}[n-m]$. Here $z^{*}$ indicates the complex conjugate of $z$. This equation is limited to a spatial interval $[-N / 2, N / 2-1]$ (the PWD's window), allowing information to be extracted locally. By scanning the image with a 1-D window of $N$ pixels, i.e., by shifting the window to all possible positions over the image, the full pixelwise PWD of the image is produced. The window can be tilted in any direction to obtain a directional distribution. Normalizing and associating $W_{z}[n, k]$ with $\breve{P}[n, k]$ in Eq. (6), the pixelwise information of the image can be extracted as follows: Let us consider a discrete sequence $z[n]$. A PWD, $w_{n}[k]$, can be calculated with $N$ data values centered at position $n$ by means of Eq. (7) and assigned to each position $n$. This local PWD can be normalized using quantum normalization as described in Subsection 2.A. This normalization identifies the PWD with a probability distribution $\breve{P}_{n}$, and the Rényi entropy associated to position $n$ can be computed as

$$
R_{3}[n]=-\frac{1}{2} \log _{2}\left(\sum_{k=1}^{N} \breve{P}_{n}^{3}[k]\right) .
$$

\section{JUSTIFICATION AND DESCRIPTION OF THE METHOD}

Anisotropy is by definition the property of being directionally dependent and is one of the topics considered by some researchers to find the relationship between the operational structure of the visual system and the natural world's content. A directional common pattern for all natural scenes cannot be determined because natural scene composition varies, and the directional content differs from sample to sample. Studies carried out by Li et al. [26] indicate that neurons tuned to horizontal are more prevalent than vertical. Natural scene content has also been the subject of anisotropic examination [27-30]. The relationship between the prevalence of natural scene content at different orientations and the visual ability to detect oriented natural scene content has been recently studied by Hansen and Essock [31]. Keil and Cristóbal [32] conducted a systematic comparison between the content biases at horizontal and vertical as a function of the spatial frequency and found greater horizontal bias at certain spatial frequencies with a preponderance of vertical content at other spatial frequencies. The experiments carried out by Keil and Cristóbal and the differences they found in the distribution of energy for natural images in the spatial-frequency domain suggest that entropic measures such as the Rényi entropy, based on measuring the frequency content of images through a directional PWD, will be an appropriate tool for image anisotropic measures. 
Natural images can be considered to be constructed by textures and edges. Generally speaking, any single image shows a diversity of textures. Such diversity of textures and edges is the origin of the anisotropy of images. Entropy can be locally measured through the spatialfrequency content of the image in a directional scheme. Hence, anisotropy is really another kind of information that may be the subject of entropic measures. Entropy measures information. Hence, differently oriented measures will provide different values of entropy according to the anisotropy of the images. Natural images can be considered random processes. Consequently, anisotropy tends to statistically cancel out, and images should be isotropic on average if their size were infinite. However, image processing techniques and the HVS handle sizelimited images. This spatial limitation of the images gives significance to slight variations in the anisotropy, which become greater as the spot is smaller and relevant when entropy is considered at the pixel level. These entropy differences are fundamentally due to the large influence of edges in the values of entropy, added to the impossibility of statistically canceling out the anisotropy of edges due to the limited size of the images. (Consider, for example, a forest with many vertical components due to the stems of the trees; here the horizontal component of the entropy will be unbalanced with the vertical component.) Edges are the origin of the main differences of image entropy when it is analyzed at the pixel level.

To formalize the calculations required to measure the anisotropy of the images, Eq. (8) will be used. This equation provides a value of entropy $R_{3}\left[n, \theta_{s}\right]$ for each pixel. Here $\theta_{s} \in\left[\theta_{1}, \theta_{2}, \ldots, \theta_{S}\right]$ represents $S$ different orientations taken to measure entropy. To define a figure of merit for the image, the expected value of this equation is calculated as

$$
\bar{R}\left[t, \theta_{s}\right]=\sum_{n} R_{3}\left[n, \theta_{s}\right] / M
$$

where $M$ represents the image size and $t \in[1,2, \ldots, T]$ is introduced to take into account the $T$ different images integrating the data set $[t$ has been omitted in the right side of Eq. (9) for simplicity]. In all the experiments described later, the expected value of the entropy for all images of the test set have been measured using six equally spaced predefined directions $\left(0^{\circ}, 30^{\circ}, 60^{\circ}, 90^{\circ}, 120^{\circ}\right.$, and $\left.150^{\circ}\right)$, although the number of such directions seems to not be critical in the method's performance. Then the variance (standard deviation or range) of these expected values has been selected as an indicator of the anisotropy of the images.

Suppose that $\bar{R}\left(t, \theta_{s}\right)$ is the expected value of entropy for image $t \in[1,2, \ldots, M]$, measured in directions $\theta_{s}$ $\in\left[\theta_{1}, \theta_{2}, \ldots, \theta_{S}\right]$. Then, the standard deviation for the resulting set of values, relative to image $t$, can be defined as

$$
\sigma(t)=\sqrt{\sum_{s=1}^{S}\left(\mu_{t}-\bar{R}\left(t, \theta_{s}\right)\right)^{2} / S},
$$

where $\mu_{t}$ is the mean of the values $\bar{R}\left(t, \theta_{s}\right)$, as defined by the expression

$$
\mu_{t}=\sum_{s=1}^{S} \bar{R}\left(t, \theta_{s}\right) / S
$$

and the range in image $t$ can be defined as

$$
r g(t)=\max \left\{\bar{R}\left(t, \theta_{s}\right)\right\}-\min \left\{\bar{R}\left(t, \theta_{s}\right)\right\}
$$

To study the statistical performance of the entropy distributions of natural images, a set of 36 images of 256 $\times 256$ pixels and 8 bits/pixel have been randomly selected and processed using the method described here (see Fig. 1).

First, in order to illustrate the performance of the method, a set of ten progressively blurred images has been generated from each image in Fig. 1 by iteratively applying a blurring point-spread function (PSF) to the source images (labeled as " 0 " in the example shown in Fig. 2).

The maximum blurring appears on the left-hand side of Fig. 2 (labeled as “-10"). Also, another set of ten progressively noisier images has been generated by iteratively adding a constant amount of Gaussian noise, starting from the same original image. The noisiest image is on the right-hand side of Fig. 2, labeled as "10". This constitutes a set of 21 registered versions of the same view, 20 degraded by blur or noise with different strengths plus the original reference image.

An experimental data test has been carried out, consisting in calculating the expectation value of the Rényi entropy for the 21 registered versions, using the image data set depicted in Fig. 1. The entropy has been calculated using Eqs. (8) and (9). A fixed window size of $N=8$ has been considered, and a horizontal orientation for the windows has been applied. Later, the procedure will be extended to any number of orientations. Figure 3 shows the results of the entropy values obtained from the image data set presented in Fig. 2. This experiment has been repeated for all natural images shown in Fig. 1. Remarkably, the graphic presented in Fig. 3, which takes into account the expected values of entropy in each set, has presented an analogous shape for all the 36 natural images considered in this study.

The results indicate how entropy increases when blur diminishes, but it also increases when more and more noise is added. This excludes entropy as a good indicator of quality by itself. Only if noise-free versions are compared can entropy and quality be directly correlated. These results are in agreement with other theoretical studies based on the multiresolution information analysis of images that conclude that entropy per pixel is strictly decreasing with respect to decreasing resolution [33].

Results of the quality metrics given by Eqs. (10) and (12) are shown in Fig. 4. The same procedure was applied to all images shown in Fig. 1, obtaining in all cases a similar steady response. It is remarkable to note that the results shown in Fig. 4 closely resemble the performance of an ideal image QA function [34]. The shapes of such curves present the following desirable properties as the most salient features: (i) accuracy, i.e., a distinct maximum is attained for the best quality; (ii) unimodality, i.e., it has a single maximum for the best quality; and (iii) computational efficiency. The selection of the standard de- 

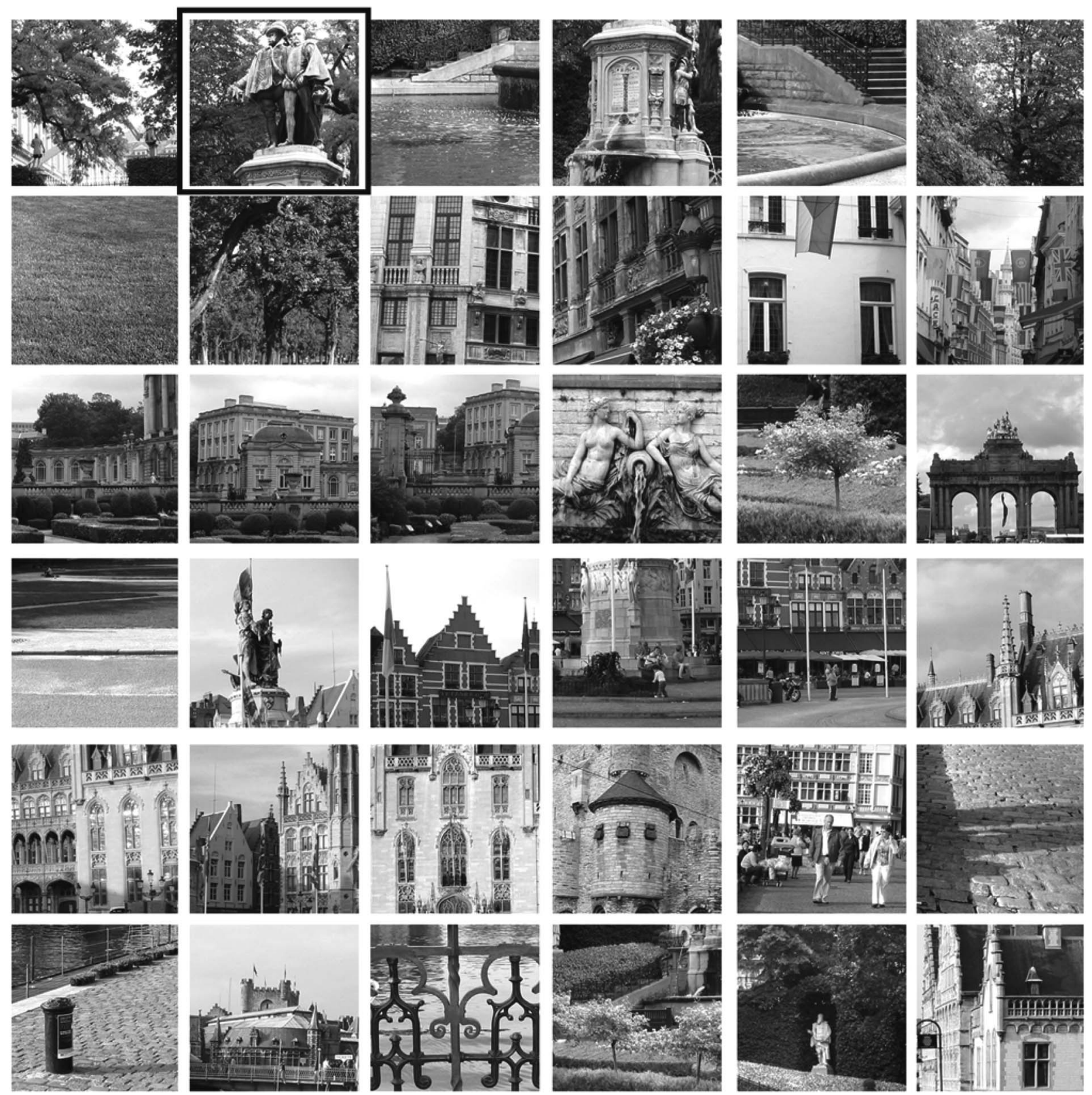

Fig. 1. Thirty-six images used for empirically determining the directional entropy in natural images. Framed image processing is described in Fig. 2 and in the text.
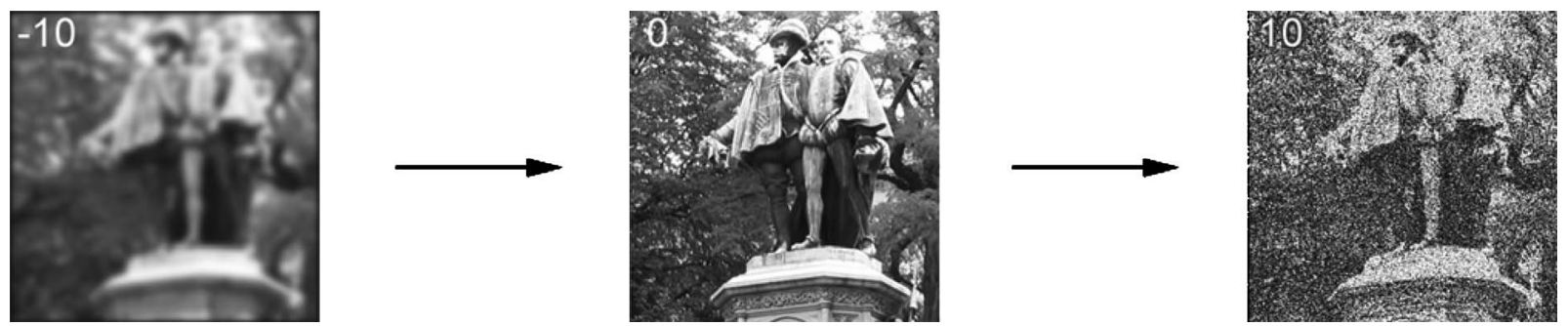

Fig. 2. Test scheme consisting in 21 degraded images. Blur decreases from -10 to 0 and noise increases from 0 to 10 . The central image is the original source image.

viation and the range of the entropy have been empirically confirmed as a good indicator of anisotropy for natural images. In-focus, noise-free natural images have shown a maximum anisotropy if compared to other degraded versions.

\section{EXPERIMENTAL RESULTS}

The method has been tested for classifying the image quality results of different algorithms. Figure 5 illustrates the results of the quality sorting provided by the 


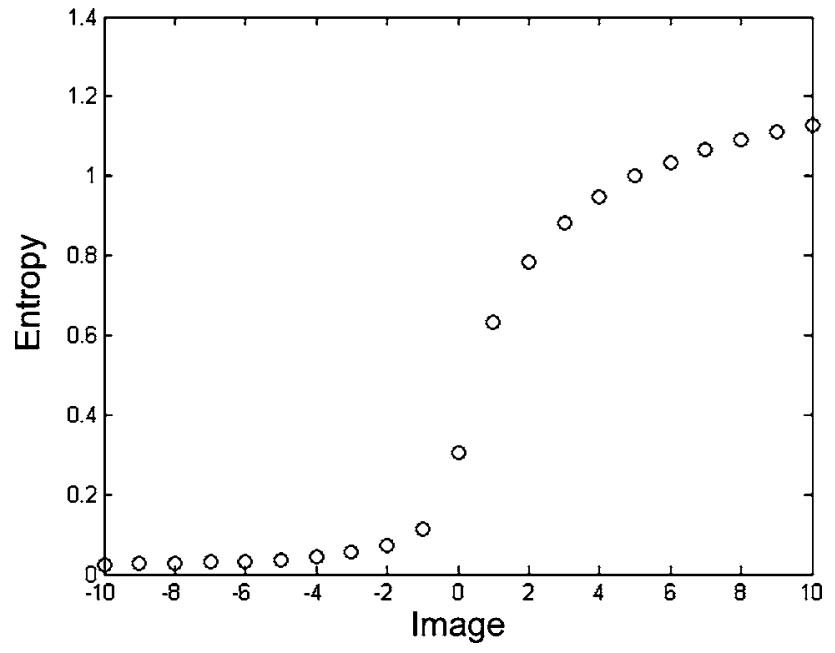

Fig. 3. Expected value of the pixelwise Rényi entropy of the 21 images of the test set presented in Fig. 2.

method for two well-known images (Lena and MIT) after the application of different denoising algorithms [35]. Images labeled as \#1 and \#7 correspond to the reference or ground truth images. In both cases the classification re-

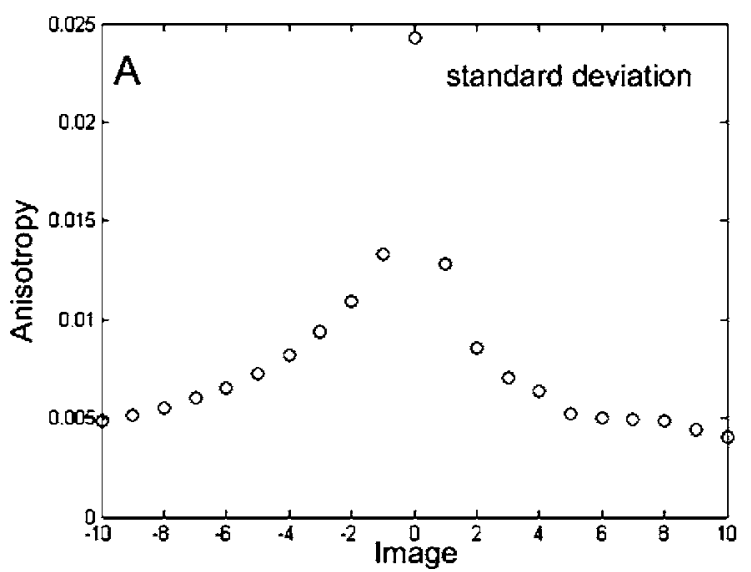

sults match well with the human visual preference and also with the PSNR. From left to right, images seem to degrade with increasing blur or noise. The standard deviation of the expected values of the Rényi directional entropy has been considered to achieve the classification, and the resulting values have been normalized between 0 and 1 in order to facilitate the visual assessment. Table 1 shows the quantitative results provided by the described method in comparison with the PSNR and the SSIM metric [3].

Similar results have been observed with other natural images (not shown here). Nevertheless, images to be classified by this method must fulfill some requirements in order to guarantee the reliability of the measurement. Images to be classified must be registered, and degradation must be uniform. To illustrate this assumption, another example in the area of superresolution (SR) imaging [36] is shown in Fig. 6. The images shown in Fig. 6(a) present a spatial-variant blur as two 3-D objects compete to be in focus at the same time. Hence, as the images are 2-D representations of $3-\mathrm{D}$ objects, different areas in the same image may suffer from different amounts of degradation. Bearing in mind that in this method the quality is measured as an average value, classification cannot be com-

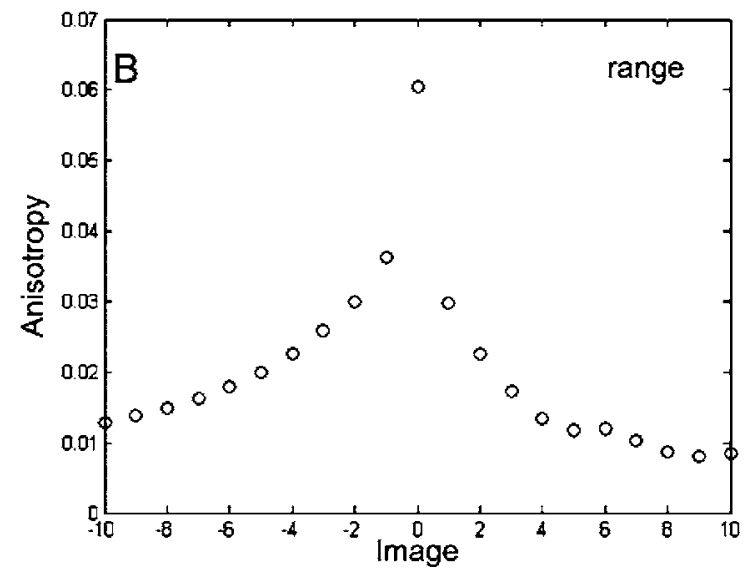

Fig. 4. A. Standard deviation of the expected values of the Rényi directional entropy for the images shown in Fig. 2. B. Range of the expected values of the Rényi directional entropy for the images in Fig. 2 . The variability refers to six different equally spaced orientations of the entropy in the image. The maximum variability corresponds to the original image, as an in-focus, noise-free version of the test set defined in Fig. 2.

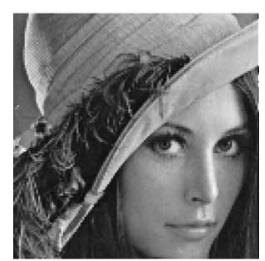

\#1

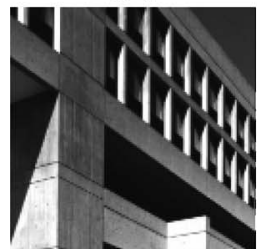

\#7

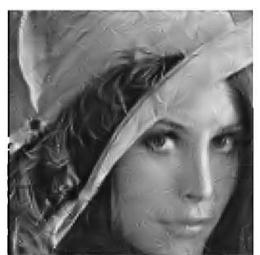

\#2

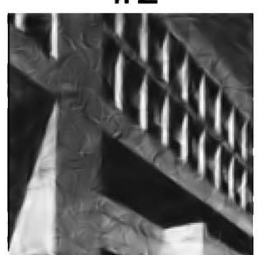

\#8

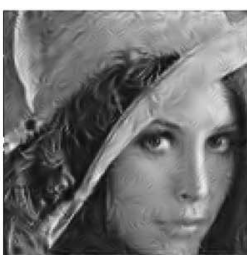

\#3

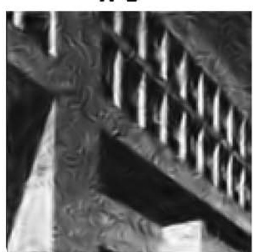

\#9

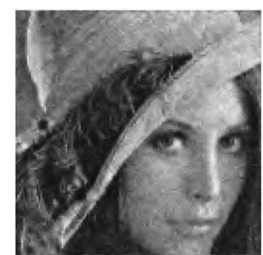

\#4

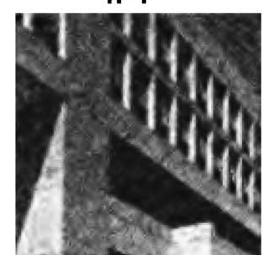

\#10

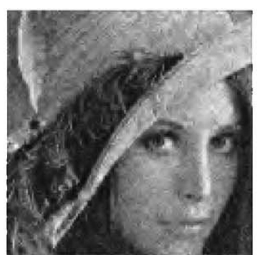

\#5

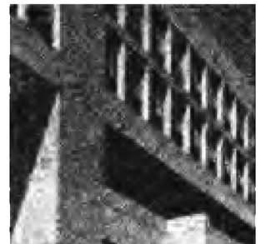

\#11

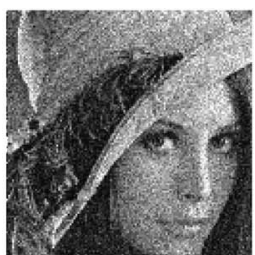

\#6

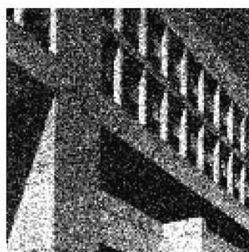

\#12

Fig. 5. Upper row (from left to right): original Lena image (\#1) and progressively degraded blurred and noisy versions. Bottom row (from left to right): original MIT image (\#7) and progressively degraded blurred and noisy versions. Images are courtesy of Sylvain Fischer [35]. 
Table 1. Comparison of Different Image Quality Measures

\begin{tabular}{llllllll}
\hline Lena & PSNR & SSIM & $\sigma(t)$ & MIT & PSNR & SSIM & $\sigma(t)$ \\
\hline$\# 1$ & - & 1 & 1 & $\# 7$ & - & 1 & 1 \\
$\# 2$ & 26.01 & 0.7923 & 0.82 & $\# 8$ & 21.77 & 0.6618 & 0.77 \\
$\# 3$ & 25.51 & 0.7766 & 0.80 & $\# 9$ & 21.24 & 0.6273 & 0.74 \\
$\# 4$ & 24.99 & 0.7459 & 0.72 & $\# 10$ & 20.57 & 0.5917 & 0.66 \\
$\# 5$ & 24.36 & 0.7129 & 0.71 & $\# 11$ & 20.00 & 0.5574 & 0.65 \\
$\# 6$ & 20.34 & 0.5357 & 0.55 & $\# 12$ & 15.56 & 0.4002 & 0.39 \\
\hline
\end{tabular}

pletely satisfactory; i.e., some areas are in focus while other areas are out of focus in the same photogram. Hence, on average, images are well classified but, in separate regions (the face of the child, the shirt, the head in the foreground, etc.) some disagreement with the visual appearance is possible. Figure 6(b) shows another example in the same SR area where the sorted quality of the images correlates well with the visual judgment. In this application domain (as in the case of image fusion) it is very common that the reference image is absent, hindering the assessment of the different results.

In order to determine whether this method is in agreement with human judgment, we have also conducted a set of experiments with a group of four images taken from the LIVE database [37] proposed by Sheikh et al. [38], which can be considered to be good representatives of natural and artificial images. In [38] they present the results of an extensive subjective QA study in which a total of 779 distorted images were evaluated by human subjects, and they use the results to evaluate the performance of several prominent full-reference image QA algorithms. Tables 2-5 present and compare the results obtained with a group of four images (the luminance component only) extracted from the above-mentioned database, after measuring their quality by means of the method described here. It is necessary to remark on the good performance of the current method for smooth distortion (blur) and random noise (see Tables 2 and 3). Structured distortion such as in the case of JPEG compression presents special arrangements of equal-valued pixels along privileged directions (horizontal and vertical). Blocking artifacts can be artificially introduced by the JPEG compression procedure, which can produce an erroneous estimate of the anisotropy measure. A feasible solution for reducing the blocking artifacts can be based on applying a deblocking filtering as it was proposed in [39]. The second approach is to consider that structured geometrical distortion increases the anisotropy of JPEG images due to the presence of zero-entropy values in the histogram. The current method is initially intended to be used for the QA of natural images. However, a slight modification of this measure will allow its application to JPEG compressed images. The modification is based on introducing a correcting factor that accounts for the number of zero-entropy values in the directional entropy histograms. This factor penalizes the presence of geometrically equal-valued pixels that are responsible for the high zero-entropy counts in JPEG images. A high zero-entropy count rarely appears in the entropy histograms of natural images. Therefore such criterion can be used when natural and JPEG images are compared. Suppose that $L$ is the size of the image and $S$ is the number of directions used to measure the image's anisotropy. Suppose also that $K$ is the total number of zeroentropy values found in the $S$ orientations while measuring the entropy. Then

$$
\widetilde{\sigma}=\sigma\left(1-\left(\frac{K}{S L}\right)^{\beta}\right)
$$

provides a corrected measure of anisotropy for JPEG images. In Eq. (13) $\sigma$ is given by Eq. (10) and $\beta$ is a parameter that penalizes the strength of the zero-entropy counts over the original anisotropy measure and whose value has been empirically determined to be $\beta=0.1$. Table

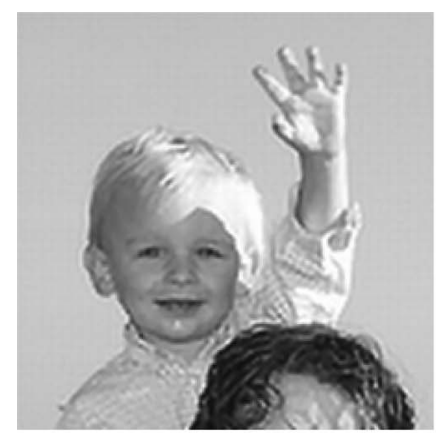

1.00

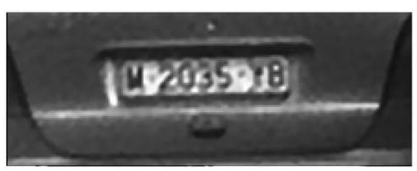

1.00

(a)
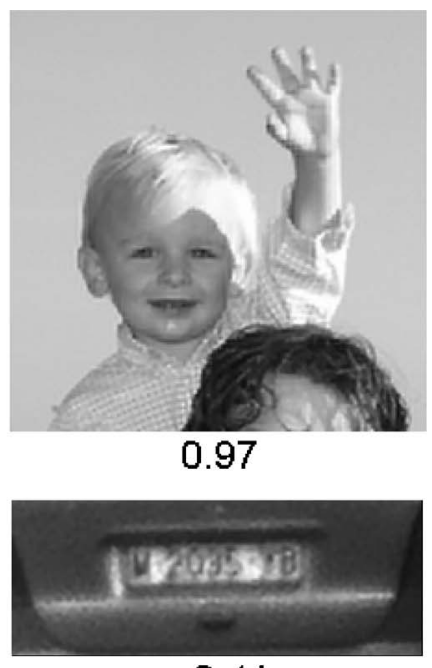

0.41

(b)

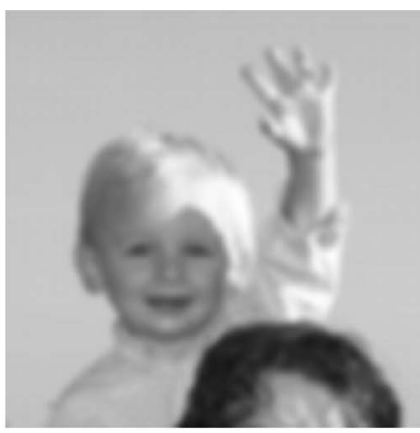

0.35

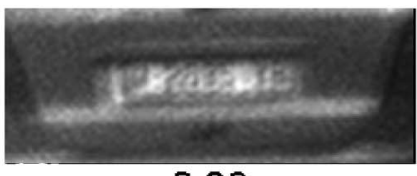

0.39

(c)

Fig. 6. Classification obtained with two sets of test images in a superresolution scenario. From left to right, image quality decreases and a quantitative figure of merit (standard deviation of directional entropy per pixel) is given at the bottom of each image. Images are courtesy of Filip Sroubek. 
Table 2. Algorithm Evaluation Using the Four Images ${ }^{a}$

\begin{tabular}{|c|c|c|c|c|c|c|c|}
\hline Buildings & $\begin{array}{c}\sigma(k) / \\
(\mathrm{PSNR})\end{array}$ & Lighthouse & $\begin{array}{c}\sigma(k) / \\
(\mathrm{PSNR})\end{array}$ & Statue & $\begin{array}{c}\sigma(k) / \\
(\mathrm{PSNR})\end{array}$ & Stream & $\begin{array}{c}\sigma(k) / \\
(\mathrm{PSNR})\end{array}$ \\
\hline $\begin{array}{l}\# 159 \\
(0.000)\end{array}$ & 1 & $\begin{array}{l}\# 164 \\
(0.00)\end{array}$ & 1 & $\begin{array}{c}\# 148 \\
(0.000)\end{array}$ & 1 & $\begin{array}{c}\# 151 \\
(0.000)\end{array}$ & 1 \\
\hline $\begin{array}{l}\# 45 \\
(0.5624)\end{array}$ & $\begin{array}{c}\mathbf{0 . 7 1} \\
(29.41)\end{array}$ & $\begin{array}{c}\# 4 \\
(0.4478)\end{array}$ & $\begin{array}{c}\mathbf{0 . 8 2} \\
(39.15)\end{array}$ & $\begin{array}{c}\# 98 \\
(0.8489)\end{array}$ & $\begin{array}{c}\mathbf{0 . 5 8} \\
(31.38)\end{array}$ & $\begin{array}{c}\# 71 \\
(0.4192)\end{array}$ & $\begin{array}{c}\mathbf{0 . 9 0} \\
(37.4)\end{array}$ \\
\hline $\begin{array}{l}\# 7 \\
(0.8489)\end{array}$ & $\begin{array}{c}\mathbf{0 . 4 9} \\
(24.29)\end{array}$ & $\begin{array}{c}\# 102 \\
(0.8220)\end{array}$ & $\begin{array}{c}\mathbf{0 . 4 6} \\
(28.32)\end{array}$ & $\begin{array}{c}\# 77 \\
(1.3072)\end{array}$ & $\begin{array}{c}\mathbf{0 . 4 1} \\
(28.6)\end{array}$ & $\begin{array}{c}\# 18 \\
(0.7629)\end{array}$ & $\begin{array}{c}\mathbf{0 . 5 5} \\
(25.29)\end{array}$ \\
\hline $\begin{array}{l}\# 62 \\
(0.9348)\end{array}$ & $\begin{array}{c}\mathbf{0 . 4 5} \\
(23.52)\end{array}$ & $\begin{array}{c}\# 15 \\
(1.1353)\end{array}$ & $\begin{array}{c}\mathbf{0 . 3 2} \\
(25.93)\end{array}$ & $\begin{array}{c}\# 131 \\
(1.8228)\end{array}$ & $\begin{array}{c}\mathbf{0 . 2 8} \\
(26.98)\end{array}$ & $\begin{array}{c}\# 126 \\
(0.834)\end{array}$ & $\begin{array}{c}\mathbf{0 . 5 1} \\
(24.55)\end{array}$ \\
\hline $\begin{array}{l}\# 134 \\
(1.5364)\end{array}$ & $\begin{array}{c}\mathbf{0 . 2 4} \\
(20.62)\end{array}$ & $\begin{array}{c}\# 97 \\
(1.4791)\end{array}$ & $\begin{array}{c}\mathbf{0 . 2 3} \\
(24.5)\end{array}$ & $\begin{array}{c}\# 54 \\
(2.166)\end{array}$ & $\begin{array}{c}\mathbf{0 . 2 2} \\
(26.24)\end{array}$ & $\begin{array}{c}\# 50 \\
(1.020)\end{array}$ & $\begin{array}{c}\mathbf{0 . 4 2} \\
(23.23)\end{array}$ \\
\hline $\begin{array}{l}\# 73 \\
(2.6249)\end{array}$ & $\begin{array}{c}\mathbf{0 . 0 7} \\
(18.64)\end{array}$ & $\begin{array}{c}\# 24 \\
(14.999)\end{array}$ & $\begin{array}{l}\mathbf{0 . 0 0 3} \\
(18.5)\end{array}$ & $\begin{array}{c}\# 120 \\
(3.999)\end{array}$ & $\begin{array}{c}\mathbf{0 . 0 5} \\
(24.02)\end{array}$ & $\begin{array}{c}\# 58 \\
(3.0833)\end{array}$ & $\begin{array}{c}\mathbf{0 . 0 7} \\
(19.57)\end{array}$ \\
\hline
\end{tabular}

a“"Buildings," "Lighthouse," "Statue," and "Stream" taken from the LIVE database for BLUR degradation. The standard deviation of the Gaussian kernel is indicated below the reference number of each image (columns 1,3,5, and 7). The corresponding PSNR has been included in columns 2, 4, 6, and 8.

Table 3. Algorithm Evaluation Using the Four Images ${ }^{a}$

\begin{tabular}{|c|c|c|c|c|c|c|c|}
\hline Buildings & $\begin{array}{c}\sigma(k) / \\
(\mathrm{PSNR})\end{array}$ & Lighthouse & $\begin{array}{c}\sigma(k) / \\
(\mathrm{PSNR})\end{array}$ & Statue & $\begin{array}{c}\sigma(k) / \\
(\mathrm{PSNR})\end{array}$ & Stream & $\begin{array}{c}\sigma(k) / \\
(\mathrm{PSNR})\end{array}$ \\
\hline $\begin{array}{l}\# 159 \\
(0.000)\end{array}$ & 1 & $\begin{array}{l}\# 164 \\
(0.00)\end{array}$ & 1 & $\begin{array}{c}\# 148 \\
(0.000)\end{array}$ & 1 & $\begin{array}{c}\# 151 \\
(0.000)\end{array}$ & 1 \\
\hline $\begin{array}{l}\# 103 \\
(0.031)\end{array}$ & $\begin{array}{c}\mathbf{0 . 9 9} \\
(33.61)\end{array}$ & $\begin{array}{c}\# 43 \\
(0.019)\end{array}$ & $\begin{array}{c}\mathbf{0 . 9 9} \\
(37.56)\end{array}$ & $\begin{array}{c}\# 85 \\
(0.015)\end{array}$ & $\begin{array}{c}\mathbf{0 . 9 6} \\
(39.46)\end{array}$ & $\begin{array}{c}\# 138 \\
(0.031)\end{array}$ & $\begin{array}{c}\mathbf{0 . 9 8} \\
(33.6)\end{array}$ \\
\hline $\begin{array}{l}\# 46 \\
(0.058)\end{array}$ & $\begin{array}{c}\mathbf{0 . 9 4} \\
(28.24)\end{array}$ & $\begin{array}{c}\# 114 \\
(0.039)\end{array}$ & $\begin{array}{c}\mathbf{0 . 9 7} \\
(31.63)\end{array}$ & $\begin{array}{c}\# 55 \\
(0.046)\end{array}$ & $\begin{array}{c}\mathbf{0 . 8 2} \\
(30.2)\end{array}$ & $\begin{array}{c}\# 88 \\
(0.062)\end{array}$ & $\begin{array}{c}\mathbf{0 . 9 3} \\
(27.63)\end{array}$ \\
\hline $\begin{array}{l}\# 130 \\
(0.2890)\end{array}$ & $\begin{array}{c}\mathbf{0 . 4 0} \\
(15.49)\end{array}$ & $\begin{array}{c}\# 40 \\
(0.062)\end{array}$ & $\begin{array}{c}\mathbf{0 . 9 3} \\
(27.58)\end{array}$ & $\begin{array}{c}\# 25 \\
(0.109)\end{array}$ & $\begin{array}{c}\mathbf{0 . 5 6} \\
(23.12)\end{array}$ & $\begin{array}{c}\# 2 \\
(0.187)\end{array}$ & $\begin{array}{c}\mathbf{0 . 6 3} \\
(18.48)\end{array}$ \\
\hline $\begin{array}{l}\# 61 \\
(0.4062)\end{array}$ & $\begin{array}{c}\mathbf{0 . 2 6} \\
(13.38)\end{array}$ & $\begin{array}{c}\# 96 \\
(0.171)\end{array}$ & $\begin{array}{c}\mathbf{0 . 6 9} \\
(19.08)\end{array}$ & $\begin{array}{c}\# 91 \\
(0.203)\end{array}$ & $\begin{array}{c}\mathbf{0 . 3 8} \\
(18.14)\end{array}$ & $\begin{array}{c}\# 106 \\
(0.312)\end{array}$ & $\begin{array}{c}\mathbf{0 . 3 6} \\
(14.86)\end{array}$ \\
\hline $\begin{array}{l}\# 4 \\
(1.9960)\end{array}$ & $\begin{array}{c}\mathbf{0 . 0 3} \\
(8.65)\end{array}$ & $\begin{array}{c}\# 66 \\
(1.000)\end{array}$ & $\begin{array}{c}\mathbf{0 . 1 2} \\
(10.19)\end{array}$ & $\begin{array}{l}\# 145 \\
(1.00)\end{array}$ & $\begin{array}{c}\mathbf{0 . 0 5} \\
(9.63)\end{array}$ & $\begin{array}{c}\# 131 \\
(0.500)\end{array}$ & $\begin{array}{c}\mathbf{0 . 2 1} \\
(12.28)\end{array}$ \\
\hline
\end{tabular}

a"Buildings," "Lighthouse," "Statue," and "Stream" taken from the LIVE database for WHITE NOISE degradation. The standard deviation of the noise is indicated below the reference number of each image (columns 1,3,5, and 7). The corresponding PSNR has been included in columns 2, 4, 6, and 8.

4 shows how the corrected metric successfully discounts the blocking artifact in the case of JPEG compressed images and provides a good sorting estimate of their quality.

Table 4. Algorithm Evaluation Using the Four Images $^{a}$

\begin{tabular}{llclcccc}
\hline Buildings & $\sigma(k)$ & Lighthouse & $\sigma(k)$ & Statue & $\sigma(k)$ & Stream & $\sigma(k)$ \\
\hline$\# 157$ & $\mathbf{1}$ & $\# 3$ & $\mathbf{1}$ & $\# 13$ & $\mathbf{1}$ & $\# 212$ & $\mathbf{1}$ \\
$(1.77)$ & & $(0)$ & & $(2.77)$ & & $(0)$ & \\
$\# 227$ & $\mathbf{0 . 8 6}$ & $\# 57$ & $\mathbf{0 . 8 8}$ & $\# 70$ & $\mathbf{0 . 9 4}$ & $\# 137$ & $\mathbf{0 . 8 5}$ \\
$(0)$ & & $(2.6)$ & & $(2.19)$ & & $(1.683)$ & \\
$\# 163$ & $\mathbf{0 . 7 1}$ & $\# 231$ & $\mathbf{0 . 6 4}$ & $\# 130$ & $\mathbf{0 . 8 9}$ & $\# 185$ & $\mathbf{0 . 7 3}$ \\
$(1.03)$ & & $(1.29)$ & & $(1.10)$ & & $(1.00)$ & \\
$\# 43$ & $\mathbf{0 . 4 5}$ & $\# 44$ & $\mathbf{0 . 2 9}$ & $\# 208$ & $\mathbf{0 . 8 4}$ & $\# 16$ & $\mathbf{0 . 6 3}$ \\
$(0.58)$ & & $(0.42)$ & & $(0)$ & & $(0.57)$ & \\
$\# 162$ & $\mathbf{0 . 3 5}$ & $\# 86$ & $\mathbf{0 . 2 6}$ & $\# 221$ & $\mathbf{0 . 7 1}$ & $\# 85$ & $\mathbf{0 . 5 7}$ \\
$(0.267)$ & & $(0.39)$ & & $(0.165)$ & & $(0.41)$ & \\
$\# 204$ & $\mathbf{0 . 3 3}$ & $\# 161$ & $\mathbf{0 . 1 7}$ & $\# 11$ & $\mathbf{0 . 6 8}$ & $\# 100$ & $\mathbf{0 . 5 1}$ \\
$(0.247)$ & & $(0.19)$ & & $(0.29)$ & & $(0.29)$ & \\
& & $\# 131$ & $\mathbf{0 . 1 6}$ & & & $\# 217$ & $\mathbf{0 . 3 6}$ \\
& & $(0.18)$ & & & & $(0.20)$ & \\
\hline
\end{tabular}

a"Buildings," "Lighthouse," "Statue," and "Stream" taken from the LIVE database for JPEG compression. The compression bitrate is indicated below the reference number of each image (columns 1,3,5, and 7).
The current procedure for the tested data set produces a good match with human judgment, although the authors recognize that extensive testing with ground truth data is required for this method to be widely accepted as a QA metric. This work has concentrated on the development of a new objective quality metric, and we used the LIVE database as a reference for the subjective assessment with human subjects. Further work will explore the influence of the combined presence of multiple artifacts such as blockiness and noisiness.

\section{CONCLUSIONS}

A new method of image QA has been introduced in this paper. The new metric provides an image quality measure without a reference or ground truth image, facilitating in general a sorting mechanism for selecting the best image among a set of processed images. Besides that, a distinct feature of this measure is that it is capable of distinguishing the presence of noise in images by decreasing its value when noise is present. The method is based on measuring the averaged anisotropy of the image by means of a pixelwise directional entropy. The robustness of the method has been experimentally validated in the case of natural 
Table 5. Algorithm Evaluation Using the Four Images ${ }^{a}$

\begin{tabular}{|c|c|c|c|c|c|c|c|}
\hline Buildings & $\sigma(k)$ & Lighthouse & $\sigma(k)$ & Statue & $\sigma(k)$ & Stream & $\sigma(k)$ \\
\hline $\begin{array}{l}\# 199 \\
(1.666)\end{array}$ & 1 & $\begin{array}{c}\# 174 \\
(1.54)\end{array}$ & 1 & $\begin{array}{l}\# 162 \\
(0.74)\end{array}$ & 1 & $\begin{array}{l}\# 217 \\
(1.48)\end{array}$ & 1 \\
\hline $\begin{array}{l}\# 222 \\
(0.84)\end{array}$ & 0.96 & $\begin{array}{c}\# 51 \\
(0.6505)\end{array}$ & 0.97 & $\begin{array}{c}\# 102 \\
(2.41)\end{array}$ & 0.99 & $\begin{array}{c}\# 17 \\
(0.40)\end{array}$ & 0.98 \\
\hline $\begin{array}{l}\# 13 \\
(0.40)\end{array}$ & 0.89 & $\begin{array}{c}\# 149 \\
(0.364)\end{array}$ & 0.96 & $\begin{array}{c}\# 116 \\
(0.222)\end{array}$ & 0.97 & $\begin{array}{c}\# 8 \\
(0.71)\end{array}$ & 0.93 \\
\hline $\begin{array}{l}\# 33 \\
(0.37)\end{array}$ & 0.88 & $\begin{array}{c}\# 106 \\
(0.242)\end{array}$ & 0.92 & $\begin{array}{c}\# 227 \\
(0.05)\end{array}$ & 0.97 & $\begin{array}{c}\# 71 \\
(0.19)\end{array}$ & 0.79 \\
\hline $\begin{array}{l}\# 29 \\
(0.20)\end{array}$ & 0.79 & $\begin{array}{c}\# 91 \\
(0.242)\end{array}$ & 0.92 & $\begin{array}{c}\# 92 \\
(0.377)\end{array}$ & 0.95 & $\begin{array}{c}\# 175 \\
(0.050)\end{array}$ & 0.58 \\
\hline $\begin{array}{l}\# 156 \\
(0.12)\end{array}$ & 0.69 & $\begin{array}{l}\# 202 \\
(0.05)\end{array}$ & 0.75 & $\begin{array}{l}\# 169 \\
(0.07)\end{array}$ & 0.85 & & \\
\hline
\end{tabular}

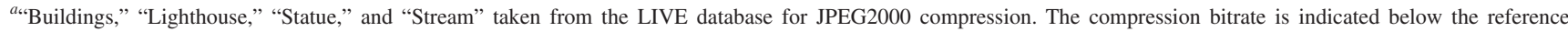
number of each image (columns $1,3,5$, and 7).

images, and results have been used to determine the relative quality of diverse processed images after applying different image processing algorithms. The experimental evaluation of the data set used demonstrates the effectiveness and promise of the proposed technique, although some assumptions such as registration and spatialinvariant degradation should be fulfilled by the images under scrutiny to obtain the best results.

\section{ACKNOWLEDGMENTS}

This research has been supported by the following projects: TEC2004-00834, TEC2005-24739-E, TEC200524046-E, and 20045OE184 from the Spanish Ministry of Education and Science and PI040765 from the Spanish Ministry of Health. We thank S. Fischer and F. Sroubek for facilitating use of the pictures of Figs. 5 and 6, respectively.

\section{REFERENCES}

1. Z. Wang and A. Bovik, "Why is image quality assessment so difficult?" IEEE International Conference on Acoustics Speech and Signal Processing (IEEE, 2002), pp. 3313-3316.

2. Z. Zhang and R. S. Blum, "A categorization of multiscaledecomposition-based image fusion schemes with a performance study for a digital camera application," Proc. IEEE 87, 1315-1328 (1999).

3. Z. Wang and A. C. Bovik, "A universal image quality index," IEEE Signal Process. Lett. 9, 81-84 (2002).

4. Z. Wang, A. C. Bovik, H. R. Sheikh, and E. P. Simoncelli, "Image quality assessment: from error visibility to structural similarity," IEEE Trans. Image Process. 13, 600-612 (2004).

5. H. R. Sheikh, A. C. Bovik, and G. DeVeciana, "An information fidelity criterion for image quality assessment using natural scene statistics," IEEE Trans. Image Process. 14, 2117-2128 (2005).

6. H. R. Sheikh and A. C. Bovik, "Image information and visual quality," IEEE Trans. Image Process. 15, 430-444 (2006).

7. H. R. Sheikh, A. C. Bovik, and L. K. Cormack, "Noreference quality assessment using natural scene statistics: JPEG2000," IEEE Trans. Image Process. 14, 1918-1927 (2005).

8. C. E. Shannon and W. Weaver, The Mathematical Theory of Communication (University of Illinois Press, 1949).

9. E. N. Kirsanova and M. G. Sadovsky, "Entropy approach in the analysis of anisotropy of digital images," Open Syst. Inf. Dyn. 9, 239-250 (2002).

10. W. J. Williams, M. L. Brown, and A. O. Hero, "Uncertainity, information and time-frequency distributions," Proc. SPIE 1566, 144-156 (1991).

11. P. Marziliano, F. Dufaux, S. Winkler, and T. Ebrahimi, "Perceptual blur and ringing metrics: application to JPEG2000," Signal Process. 19, 163-172 (2004).

12. N. Cvejic, C. N. Canagarajah, and D. R. Bull, "Image fusion metric based on mutual information and Tsallis entropy," Electron. Lett. 42, 626-627 (2006).

13. C. S. Xydeas and V. Petkovic, "Objective image fusion performance measure," Electron. Lett. 36, 308-309 (2000).

14. G. Qu, D. Zhang, and P. Yang, "Information measure for performance of image fusion," Electron. Lett. 38, 313-315 (2002).

15. R. Danserau and W. Kinsner, "New relative multifractal dimension measures," in IEEE International Conference on Acoustics Speech and Signal Processing (IEEE, 2001), pp. 1741-1744.

16. L. Stankovic, "A measure of some time-frequency distributions concentration," Signal Process. 81, 621-631 (2001).

17. N. Wiener, Cybernetics (Wiley, 1948).

18. A. Rényi, "Some fundamental questions of information theory," in Selected Papers of Alfréd Rényi, Pál Turán, ed. (Akadémiai Kiadó, 1976), Vol. 3, pp. 526-552 [Originally in Magy. Tud. Akad. III Oszt. Kózl. 10, 251-282 (1960)].

19. T. H. Sang and W. J. Williams, "Rényi information and signal dependent optimal kernel design," in IEEE International Conference on Acoustics Speech and Signal Processing (IEEE, 1995), Vol. 2, pp. 997-1000.

20. P. Flandrin, R. G. Baraniuk, and O. Michel, "Time-frequency complexity and information," in IEEE International Conference on Acoustics Speech and Signal Processing (IEEE, 1994), Vol. 3, pp. 329-332.

21. R. Eisberg and R. Resnick, Quantum Physics (Wiley, 1974)

22. L. D. Jacobson and H. Wechsler, "Joint spatial/spatialfrequency representation," Signal Process. 14, 37-68 (1988).

23. E. Wigner, "On the quantum correction for thermodynamic equilibrium," Phys. Rev. 40, 749-759 (1932).

24. T. A. C. M. Claasen and W. F. G. Mecklenbräuker, "The Wigner distribution-a tool for time frequency analysis, Parts I-III," Philips J. Res. 35, 217-250, 276-300, 372-389 (1980).

25. K. H. Brenner, "A discrete version of the Wigner distribution function," in Proceedings of EURASIP, Signal Processing II: Theories and Applications (North Holland, 1983), pp. 307-309.

26. B. Li, M. R. Peterson, and R. D. Freeman, "Oblique effect: a neural bias in the visual cortex," J. Neurophysiol. 90, $204-217$ (2003). 
27. E. Switkes, M. J. Mayer, and J. A. Sloan, "Spatial frequency analysis of the visual environment: anisotropy and the carpentered environment hypothesis," Vision Res. 18, 1393-1399 (1978).

28. R. J. Baddeley and P. J. B. Hancock, "A statistical analysis of natural images matches psychophysically derived orientation tuning curves," Proc. R. Soc. London, Ser. B 246, 219-223 (1991)

29. P. J. B. Hancock, R. J. Baddeley, and L. S. Smith, "The principal components of natural images," Network Comput. Neural Syst. 3, 61-70 (1992).

30. J. Haung and D. Mumford, "Statistics of natural images and models," in Proceedings of the ICCV , 1, 541-547 (1999).

31. B. C. Hansen and E. A. Essock, "A horizontal bias in human visual processing of orientation and its correspondence to the structural components of natural scenes," J. Vision 4, 1044-1060 (2004).

32. M. S. Keil and G. Cristóbal, "Separating the chaff from the wheat: possible origins of the oblique effect," J. Opt. Soc. Am. A 17, 697-710 (2000).

33. R. Román, J. J. Quesada, and J. Martínez, "Multiresolution-information analysis for images," Signal Process. 24, 77-91 (1991).
34. Y. Qu, Z. Pu, H. Zhao, and Y. Zhao, "Comparison of different quality assessment functions in autoregulative illumination intensity algorithms," Opt. Eng. (Bellingham) 45, 117-201 (2006).

35. S. Fischer, F. Sroubek, L. Perrinet, R. Redondo, and G. Cristóbal, "Self-invertible 2D Gabor wavelets," Int. J. Comput. Vis. available at http://www.springerlink.com/ content/07q411454q407047/fulltext.pdf.

36. F. Sroubek, G. Cristóbal, and J. Flusser, "Combined superresolution and blind deconvolution," in Information Optics: 5th International Workshop (American Institute of Physics, 2006), paper CP860, pp. 15-26.

37. H. R. Sheikh, Z. Wang, L. Cormack, and A. C. Bovik, "LIVE image quality assessment database," Release 2, 2005 [Online]. Available at http://live.ece.utexas.edu/research/ quality.

38. H. R. Sheikh, M. F. Sabir, and A. C. Bovik, "A statistical evaluation of recent full reference image quality assessment algorithms," IEEE Trans. Image Process. 15, 3440-3451 (2006).

39. M. Y. Shen and C. C. Jay Kuo, "Review of postprocessing techniques for compression artifact removal," J. Visual Commun. Image Represent 9, 2-14 (1998). 